Journal for ImmunoTherapy of Cancer

\title{
KALRN mutations promote antitumor immunity and immunotherapy response in cancer
}

\author{
Mengyuan Li, ${ }^{1,2,3}$ Yuxiang Ma, ${ }^{4}$ You Zhong, ${ }^{4}$ Qian Liu, ${ }^{1,2,3}$ Canping Chen, ${ }^{1,2,3}$ \\ Lei Qiang, ${ }^{4}$ Xiaosheng Wang (D) ${ }^{1,2,3}$
}

To cite: Li M, Ma Y, Zhong Y, et al. KALRN mutations promote antitumor immunity and immunotherapy response in cancer. Journal for ImmunoTherapy of Cancer 2020;8:e000293. doi:10.1136/ jitc-2019-000293

- Additional material is published online only. To view, please visit the journal online (http://dx.doi.org/10.1136/jitc2019-000293).

$\mathrm{ML}$ and $\mathrm{YM}$ contributed equally.

$\mathrm{ML}$ and $\mathrm{YM}$ are joint first authors.

Accepted 10 September 2020

Check for updates

(c) Author(s) (or their employer(s)) 2020. Re-use permitted under CC BY-NC. No commercial re-use. See rights and permissions. Published by BMJ.

${ }^{1}$ Biomedical Informatics Research Lab,School of Basic Medicine and Clinical Pharmacy, China Pharmaceutical University, Nanjing, China

${ }^{2}$ Cancer Genomics Research Center, School of Basic Medicine and Clinical Pharmacy, China Pharmaceutical University, Nanjing, China

${ }^{3}$ Big Data Research Institute, China Pharmaceutical University, Nanjing, China

${ }^{4}$ Department of Basic Medicine, School of Basic Medicine and Clinical Pharmacy, China Pharmaceutical University, Nanjing, China

Correspondence to Dr Xiaosheng Wang; xiaosheng.wang@cpu.edu.cn

\section{ABSTRACT}

Background kalirin RhoGEF kinase (KALRM) is mutated in a wide range of cancers. Nevertheless, the association between KALRN mutations and the pathogenesis of cancer remains unexplored. Identification of biomarkers for cancer immunotherapy response is crucial because immunotherapies only show beneficial effects in a subset of patients with cancer.

Methods We explored the correlation between KALRN mutations and antitumor immunity in 10 cancer cohorts from The Cancer Genome Atlas program by the bioinformatics approach. Moreover, we verified the findings from the bioinformatics analysis with in vitro and in vivo experiments. We explored the correlation between KALRN mutations and immunotherapy response in five cancer cohorts receiving immune checkpoint blockade therapy. Results Antitumor immune signatures were more enriched in $K A L R N$-mutated than $K A L R N$-wildtype cancers. Moreover, KALRN mutations displayed significant correlations with increased tumor mutation burden and the microsatellite instability or DNA damage repair deficiency genomic properties, which may explain the high antitumor immunity in KALRN-mutated cancers. Also, programmed cell death 1 ligand (PD-L1) expression was markedly upregulated in $K A L R N$-mutated versus $K A L R N$-wildtype cancers. The increased antitumor immune signatures and PD-L1 expression in $K A L R N$-mutated cancers may favor the response to immune checkpoint blockade therapy in this cancer subtype, as evidenced in five cancer cohorts receiving antiprogrammed cell death protein 1 (PD-1)/PDL1/cytotoxic T-lymphocyte-associated protein 4 (CTLA-4) immunotherapy. Furthermore, the significant association between KALRN mutations and increased antitumor immunity was associated with the fact that KALRN mutations compromised the function of KALRN in targeting Rho GTPases for the regulation of DNA damage repair pathways. In vitro and in vivo experiments validated the association of KALRN deficiency with antitumor immunity and the response to immune checkpoint inhibitors.

Conclusions The KALRN mutation is a useful biomarker for predicting the response to immunotherapy in patients with cancer.

\section{BACKGROUND}

Recently, immunotherapy has achieved success in treating various cancers. ${ }^{1-4}$ In particular, immune checkpoint blockade
(ICB) methods, such as inhibition of cytotoxic T-lymphocyte-associated protein 4 (CTLA-4), programmed cell death protein 1 (PD-1), and programmed cell death 1 ligand (PD-L1), have been used as therapy for many advanced malignancies. ${ }^{5}$ Nevertheless, many patients with cancer failed to respond to immunotherapy. Numerous studies have identified specific genetic or genomic features associated with cancer immunotherapy response, such as PD-L1 expression ${ }^{6}$ and tumor mutation burden (TMB). ${ }^{7}$ Mutations of certain genes, such as $T P 53^{8}$ and $A R I D 1 A,{ }^{9}$ have been associated with cancer immunotherapy response.

Kalirin RhoGEF kinase ( $K A L R N)$ encodes a protein that activates specific Rho GTPase family members to regulate neurons and the actin cytoskeleton. ${ }^{10}$ This gene is mutated in a wide range of cancers, ${ }^{11}$ for example, melanoma, lung cancer, uterine corpus endometrial carcinoma (UCEC), glioblastoma multiforme (GBM) and colorectal cancer (COAD). Nevertheless, very few reports of the association between $K A L R N$ mutations and carcinogenesis have been published. ${ }^{13}$ In this study, we investigated the associations between KALRN mutations and antitumor immune signatures (NK cells, CD8 + T cells and immune cytolytic activity (ICA)) in 10 individual cancer types (CESC, COAD, ESCA, GBM, PRAD, READ, SARC, SKCM, STAD, and UCEC) and in them all together (the pan cancer) by analyzing The Cancer Genome Atlas (TCGA, https://cancergenome.nih. gov) cohorts (online supplemental table S1). For these cohorts, we also explored the associations between KALRN mutations and cancer immunotherapy response-related biomarkers, such as PD-L1 expression and TMB. Moreover, based on five cancer cohorts receiving immunotherapy (online supplemental table S1), we investigated 
the association between $K A L R N$ mutations and cancer immunotherapy response. We validated our findings from the bioinformatics analysis by performing in vitro experiments in four tumor cell lines (MGC803, SJSA1, SW620 and B16F10), which were associated with four of the 10 cancer types, and in vivo experiments with mouse tumor models. Our study demonstrates that KALRN mutations can promote antitumor immunity and cancer immunotherapy response and are a useful biomarker for stratifying patients with cancer responsive to immunotherapy.

\section{METHODS}

Evaluation and comparisons of immune signature enrichment scores

We calculated the enrichment score for an immune signature in a tumor sample by single-sample gene-set enrichment analysis (ssGSEA) ${ }^{15}$ of the marker genes of the immune signature. Three immune signatures were analyzed, namely NK cells (marker genes KLRC1 and KLRF1), CD8 + T cells (CD8A) and ICA (PRF1 and GZMA). ${ }^{11} 1216$ We compared the immune signature enrichment scores between two classes of samples by using the Mann-Whitney $\mathrm{U}$ test (one tailed). In addition, we compared the ratios of immune-stimulatory to immune-inhibitory signatures $(\mathrm{CD} 8+/ \mathrm{CD} 4+$ regulatory $\mathrm{T}$ cells, M1/M2 macrophages, and proinflammatory/anti-inflammatory cytokines) between two classes of samples by using the Mann-Whitney $\mathrm{U}$ test (one tailed). The ratios were the log2-transformed mean expression levels of the marker genes of immunestimulatory signatures over those of immune-inhibitory signatures. We carried out these analyzes with $\mathrm{R}$ programming software (ssGSEA scores were calculated by using the R package 'GSVA'. ${ }^{15}$ )

\section{Network analysis}

We identified the protein-protein interaction network of KALRN with the webtool STRING (V.11.0, https://stringdb.org/) by using the default settings.

\section{Logistic regression analysis}

We used logistic regression with two predictors (KALRN mutation and Rho GTPase gene mutation) to predict the immune signature scores (high (upper third) vs low (bottom third) scores) and immunotherapy response (responsive vs non-responsive), respectively. Both predictors were binary variables: 1 (mutation) or 0 (no mutation). In performing the logistic regression analyzes, we used the R function 'glm' to fit the binary model and the function ' $\mathrm{lm}$. beta' in the $\mathrm{R}$ package 'QuantPsyc' to calculate the standardized regression coefficients ( $\beta$ values).

\section{Statistical analysis}

We compared the expression levels of genes and proteins between two classes of samples by using Student's t test (two tailed). We performed the analyzes with log2-transformed values of the normalized RNA-Seq gene expression values and the downloaded normalized protein expression data. We used Fisher's exact test to examine the association between two categorical variables. All these statistical analyzes were performed in the $\mathrm{R}$ programming environment (V.4.0.2).

\section{Survival analysis}

We performed the univariate survival analyzes by using the function 'survfit' in the R package 'survival', used Kaplan-Meier survival curves to display the survival time differences, and applied the log-rank test to evaluate the significance of the survival time differences.

\section{In vitro experiments}

\section{Antibodies, reagents and cell lines}

All antibodies were used at a dilution of 1:1000 unless otherwise specified. Anti-PD-L1 (ab213524) and anti-CD8 $\alpha$ were purchased from Abcam (Burlingame, CA). GAPDH (sc32233) was purchased from Santa Cruz Biotechnology, California, USA. Anti-MSH2 (15520-1-AP) was purchased from Proteintech Group (Rosemont, Illinois, USA). AntiKALRN (BS3514) was purchased from Bioworld Technology (St. Louis, MO). PE anti-mouse TNF- $\alpha$ Antibody (catalog no. 506305), APC anti-mouse IFN- $\gamma$ Antibody (catalog no. 505809), APC anti-mouse CD366 (Tim-3) Antibody (catalog no. 134007), APC anti-mouse CD279 (PD-1) Antibody (catalog no. 135209), APC anti-mouse CD223 (LAG-3) Antibody (catalog no. 125209) were purchased from BioLegend (San Diego, CA). Dulbecco's modified Eagle's medium (12100-061), Alpha minimum essential medium (MEM) (11900024) and horse serum (16050122) were purchased from Thermo Fisher Scientific (Waltham, Massachusetts, USA). Inositol (I7508), 2-mercaptoethanol (M3148) and folic acid (F8758) were purchased from Sigma-Aldrich (St. Louis, Missouri, USA). The human cancer cell lines MGC803 (gastric cancer), SJSA1 (osteosarcoma) and SW620 (colon cancer) and the murine cell line B16F10 (melanoma) were from the American Type Culture Collection. They were cultured in a complete medium (Dulbecco's modified Eagle's medium supplemented with $10 \%$ fetal bovine serum) in a humidified incubator at $37^{\circ} \mathrm{C}$ and $5 \% \mathrm{CO}_{2}$. NK92 cells (KeyGEN BioTECH, Nanjing, China) were cultured in Alpha MEM with $2 \mathrm{mM} \mathrm{L}$-glutamine, $1.5 \mathrm{~g} / \mathrm{L}$ sodium bicarbonate, $0.2 \mathrm{mM}$ inositol, $0.1 \mathrm{mM}$ 2-mercaptoethanol, $0.02 \mathrm{mM}$ folic acid, $100-200 \mathrm{U} / \mathrm{mL}$ recombinant human IL-2 (PeproTech, Rocky Hill, New Jersey, USA), and a final concentration of $12.5 \%$ horse serum and $12.5 \%$ fetal bovine serum.

Knockdown of KALRN with small interfering RNA (siRNA)

Cancer cells were transfected with KALRN siRNA or control siRNA by using Effectene Transfection Reagent (Qiagen, Hilden, Germany, B00118) according to the manufacturer's instructions. The medium was replaced after 24 hours incubation with fresh medium, and the 
cells were maintained for a further 24 hours. The transfection efficiency was detected with quantitative PCR or Western blotting. KALRN siRNA and control siRNA were synthesized by GenePharma (Shanghai, China). Their sequences were as follows: KALRN siRNA: 1, 5'-GCUU CCACUGAAGUACCUATT-3' (sense) and 5'-UAGG UACUUCAGUGGAAGCTT-3' (antisense); 2, 5'-GCAA UCGCCCAUUGAGUAUTT-3' (sense) and 5'-AUAC UCAAUGGGCGAUUGCTT-3' (antisense); 3, 5'-GCUU CGACCUUGGACACUUTT-3' (sense) and 5'-AAGU GUCCAAGGUCGAAGCTT-3' (antisense); control siRNA: 5'-UUCUCCGAACGUGUCACGUTT-3' (sense) and 5'ACGUGACACGUUCGGAGAATT-3' (antisense).

\section{Lentivirus generation and infection}

Heteroduplexes (small hairpin RNA or shRNA) containing different 19 bp RNA segments to target different locations of the KALRN gene were selected. The heteroduplexes, supplied as 58-nucleotide oligomers, were annealed, and the downstream of the $\mathrm{U} 6$ promoter was inserted into the pLKO.1 plasmid to generate pLKO.1/ShKALRN. Recombinant and control lentiviruses were produced by transiently transfecting pLKO.1/vector and pLKO.1/ ShKALRN, respectively. The lentiviruses were transfected into $293 \mathrm{~T}$ cells, along with the packaging plasmid psPAX2 and envelope plasmid pMD2.G, by using the calcium phosphate precipitation method. After 48 hours, lentiviral particles were collected and concentrated from the supernatant by ultracentrifugation. Effective lentiviral shRNA was screened by infecting these viruses with B16F10 cells, and their inhibitory effect on KALRN expression was analyzed by Western blotting. The lentivirus containing the ShKALRN RNA target sequences and a control virus were used for the animal study. The coding strand sequence of the shRNA-encoding oligonucleotides was 5'-CCGGGTGGAGTTAATGTGCCTTGTTCTCGAGA ACAAGGCACATTAACTCCACTTTTTG-3' for KALRN.

\section{Western blotting}

Cell extracts were generated by using lysis buffer supplemented with protease inhibitor cocktail immediately before use. Proteins were denatured by addition of 6 volumes of SDS sample buffer and boiling at $100^{\circ} \mathrm{C}$ for $10 \mathrm{~min}$ and were then separated by SDS-PAGE. After electrophoresis, proteins were electrotransferred onto nitrocellulose membranes. The membranes were blocked in $5 \%$ nonfat dry milk buffer at $25^{\circ} \mathrm{C}$ for 1 hour and then incubated overnight at $4^{\circ} \mathrm{C}$ with specific antibodies. Binding of the primary antibody was detected by using peroxidaseconjugated secondary antibodies and a SuperSignal West Dura chemiluminescent substrate kit (Thermo Scientific, 34075) according to the manufacturer's instructions. The Western blotting result was semiquantified by using Image J software to measure the intensities of the bands. The band densities were normalized to the background, and the relative optical density ratios were calculated relative to the housekeeping gene GAPDH.

\section{Quantitative PCR}

Total RNA was extracted from cells and reversely transcribed to cDNA. Quantitative PCR was performed with the BioRad CFX96 Touch Real-Time PCR Detection System (BioRad, Richmond, California, USA) using iQ SYBR1 Green Supermix (BioRad, Richmond, California, USA). The threshold cycle numbers were obtained by using BioRad CFX manager software. The program for amplification was one cycle of $95^{\circ} \mathrm{C}$ for $2 \mathrm{~min}$, followed by 40 cycles of $95^{\circ} \mathrm{C}$ for $10 \mathrm{~s}, 60^{\circ} \mathrm{C}$ for $30 \mathrm{~s}$, and $72^{\circ} \mathrm{C}$ for $30 \mathrm{~s}$. The relative amount of each gene was normalized to the amount of GAPDH. The primer sequences were as follows: hKALRN: 5'-GATTGTCATCTTCAGTGA-3' (forward) and 5'-AGGACCAAGTAATTCATC-3' (reverse); hGAPDH: 5'-TGTGGGCATCAATGGATTTGG-3' (forward) and 5'-ACACCATGTATTCCGG GTCAAT-3' (reverse).

\section{Co-culture of tumor cells with NK92 cells}

A transwell chamber (Corning, New York, USA) was inserted into a six-well plate to construct a co-culture system. Tumor cells (MGC803, SJSA1, SW620 and B16F10) were seeded on the six-well plate at a density of $5 \times 10^{4}$ cells/well, and NK92 cells were seeded on the membrane (polyethylene terephthalate, pore size of $0.4 \mu \mathrm{m}$ ) of the transwell chamber at a density of $5 \times 10^{4}$ cells/chamber. Tumor cells and NK92 cells were co-cultured in a humidified incubator at $37^{\circ} \mathrm{C}$ and $5 \% \mathrm{CO}_{2}$ atmosphere for 48 hours.

\section{EdU proliferation assay}

After co-culture of the tumor cells with NK92 cells for 48 hours, the proliferation capacity of the NK92 cells was measured by an EdU (5-ethynyl-2'-deoxyuridine; Invitrogen, California, USA) proliferation assay. NK92 cells were plated in 96 -well plates with a density of $2 \times 10^{3}$ cells/ well for 24 hours. Before fixation, permeabilization and EdU staining, the cells were incubated with $10 \mu \mathrm{M} \mathrm{EdU}$ at $37^{\circ} \mathrm{C}$ for 24 hours. The cell nuclei were stained with 4',6-diamidino-2-phenylindole (DAPI) at a concentration of $1 \mu \mathrm{g} / \mathrm{mL}$ for $20 \mathrm{~min}$. The proportion of NK92 cells incorporating $\mathrm{EdU}$ was determined with fluorescence microscopy. Each assay was performed in triplicate wells.

\section{In vivo experiments}

\section{In vivo mouse models}

B16F10 tumor cells were transduced with ShCon (scramble) or ShKALRN lentivirus and selected by puromycin for 7 days. The stably transfected B16F10 tumor cells $(2 \times 106)$ were subcutaneously injected into the right flank of recipient mice after shaving the injection site. After 5 days, when the tumor volume was approximately $4-5 \mathrm{~mm}^{3}$, half of the ShCon and ShKALRN mice were treated with 100 U/L PD1/PDL1 inhibitor BMS-1 (concentration $500 \mathrm{mg} / \mathrm{mL}$; i.p.) (MCE Cat. No. HY-19991) every 3 days. The tumors were isolated from mice after 15 days. Tumor volumes did not exceed the maximum allowable size according to the LJI IACUC animal experimental protocol. The 
tumor volume was measured every 2 days after the tumor appeared on the fifth day and was calculated according to the formula: $V=1 / 2 \times$ width $^{2} \times$ length.

\section{Isolation of tumor-infiltrating lymphocytes (TILS)}

From subcutaneously established B16F10 melanomabearing mice, tumors were excised and chopped with tweezers and scissors and were then digested with $2 \mathrm{mg} / \mathrm{mL}$ collagenase (type IV, Sigma, UK) for $45 \mathrm{~min}$. Following digestion, tumors were filtered through $70 \mu \mathrm{M}$ cell strainers. The cell suspension was washed twice in culture medium by centrifugation at $1500 \mathrm{rpm}$ and $4^{\circ} \mathrm{C}$ for $10 \mathrm{~min}$. After the washing, the cells were resuspended in $6 \mathrm{~mL}$ of PBS and were layered over $3 \mathrm{~mL}$ of $30 \%-100 \%$ gradient percoll (Beijing Solarbio Science \& Technology, Beijing, China); this was followed by centrifugation at $2600 \mathrm{rpm}$ for $30 \mathrm{~min}$ at $25^{\circ} \mathrm{C}$. The enriched TILs were obtained at the interface as a thin buffy layer, were washed with PBS twice, and finally were re-suspended in FACS staining buffer for further staining procedures.

\section{Flow cytometry}

TILs were stained with CD8 and PD-1 or LAG3 (Biolegend, San Diego, California, USA) and were analyzed by flow cytometry. TILs were restimulated with cell stimulation cocktail (eBioscience, San Diego, California, USA), and expression of IFN- $\gamma$ and TNF- $\alpha$ (Biolegend) was analyzed by flow cytometry. Staining for cell surface markers was performed by incubating cells with antibody (1:100 dilution) in FACS buffer $\left(0.1 \%\right.$ BSA in PBS) for $30 \mathrm{~min}$ at $4^{\circ} \mathrm{C}$. For intracellular cytokine (IFN- $\gamma$ and TNF- $\alpha$ ) staining, surface markers were stained before fixation/permeabilization (Intracellular Fixation \& Permeabilization Buffer Set, ThermoFisher).

\section{Immunofluorescence of CD8, PD-L1 and CD49b}

Paraffin-embedded mice tumor tissue (subcutaneously established B16F10 melanoma) sections ( $3 \mu \mathrm{m}$ thick) were subjected to immunofluorescence with CD8 (Abcam), PD-L1 (Abcam), or CD49b (Abcam) primary antibodies. Before immunostaining, tumor tissue sections were deparaffinized with xylene, rehydrated and unmasked in sodium citrate buffer $(10 \mathrm{mM}, \mathrm{pH} 6.0)$, and treated with a glycine solution $(2 \mathrm{mg} / \mathrm{mL})$ to quench autofluorescence. After blockade of non-specific antibody binding sites with a solution of $1 \%$ BSA in PBS for 1 hour at $25^{\circ} \mathrm{C}$, tissue slides were incubated overnight at $4^{\circ} \mathrm{C}$ with anti-CD8, anti-PD-L1, or CD 49b rabbit primary antibodies (1:200 dilution) in blocking solution. Irrelevant isotype-matched rabbit IgG (Sigma-Aldrich) was applied in parallel to serial sections as negative controls. The following day, the immunoreactions were revealed by using Alexa Fluor-488-conjugated goat anti-rabbit IgG (for CD8 and CD49b) or Alexa Fluor-594-conjugated goat anti-rabbit IgG (for PD-L1) (Invitrogen, San Diego, California, USA) secondary antibodies (1:200 dilution) in blocking solution. The immunolabeled slides were examined with a fluorescence microscope after nuclear counterstaining with DAPI. Green, red and blue channel fluorescence images were acquired with a Leica DFC310 FX 1.4-megapixel digital color camera equipped with LAS V.3.8 software (Leica Microsystems, Wetzlar, Germany). Overlay images were reconstructed by using the freeshare Image J software.

\section{RESULTS}

KALRN mutations correlate with elevated anti-tumor immunity We found that the immune signatures (NK cells, CD8 $+\mathrm{T}$ cells and ICA) consistently displayed higher enrichment levels in $K A L R N$-mutated than KALRN-wildtype cancers in the pan cancer analysis (Mann-Whitney $\mathrm{U}$ test, one tailed, $\mathrm{p}<0.001$ ) (figure $1 \mathrm{~A}$ ). Moreover, the NK cell, CD8 + T cell, and ICA enrichment scores were significantly higher in $K A L R N$-mutated than $K A L R N$ wildtype cancers in four, five, and five individual cancer types, respectively (Mann-Whitney U test, one tailed, $\mathrm{p}<0.05$ ) (figure 1A). We compared the ratios of immune-stimulatory to immune-inhibitory signatures in the pan cancer and in individual cancer types. We found that the ratios $(\mathrm{CD} 8+/ \mathrm{CD} 4+$ regulatory T cells, M1/M2 macrophages, and proinflammatory/antiinflammatory cytokines) were significantly higher in $K A L R N$-mutated than $K A L R N$-wildtype cancers in the pan cancer and in multiple individual cancer types (Mann-Whitney U test, $\mathrm{p}<0.05$ ) (figure 1B). The EdU proliferation assay showed that NK cells co-cultured with $K A L R N$-knockdown tumor cells had a significantly stronger proliferation capacity than NK cells co-cultured with $K A L R N$-wildtype tumor cells (figure 1C). Taken together, these data suggest that KALRN mutations enhance antitumor immunity in cancer.

\section{KALRN mutations correlate with increased TMB and DNA damage repair deficiency}

The TMB has been recognized as a predictive biomarker for cancer immunotherapy response. ${ }^{7}$ We found that $K A L R N$-mutated cancers exhibited a significantly higher TMB (defined as the total count of gene somatic mutations) than KALRN-wildtype cancers in the pan cancer and in all 10 individual cancer types (Mann-Whitney $\mathrm{U}$ test, one tailed, $\mathrm{p}<0.01$ ) (figure $2 \mathrm{~A}$ ). In addition, KALRNmutated cancers displayed more predicted neoantigens ${ }^{16}$ than KALRN-wildtype cancers in the pan cancer and in five individual cancer types (Mann-Whitney $\mathrm{U}$ test, $\mathrm{p}<0.01$ ) (figure $2 \mathrm{~A}$ ).

Among the five cancer types (COAD, ESCA, READ, STAD and UCEC) with a prevalent MSI subtype, MSI-high (MSI-H) cancers had significantly higher mutation rates of KALRN than MSI-low (MSI-L) and microsatellite stable (MSS) cancers in the pan cancer and in four individual cancer types (Fisher's exact test, $\mathrm{p}<0.01$ ) (figure 2B). Moreover, we found that $K A L R N$ mutations co-occurred with mutations of DNA mismatch repair pathway genes (PMS1, PMS2, MLH1, 
A

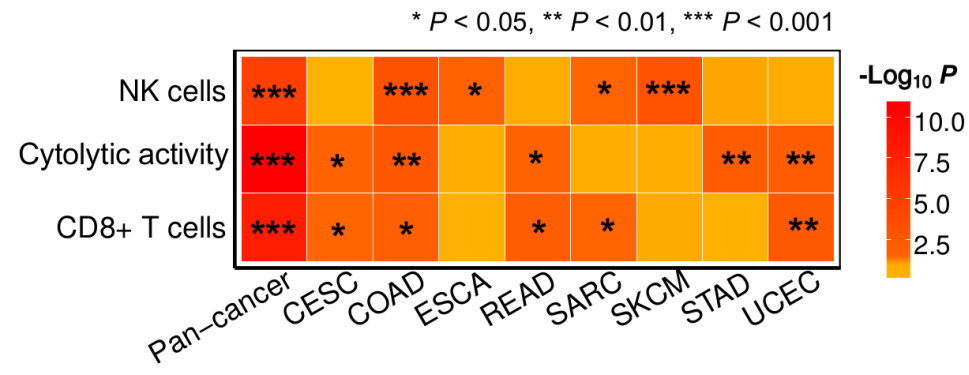

B

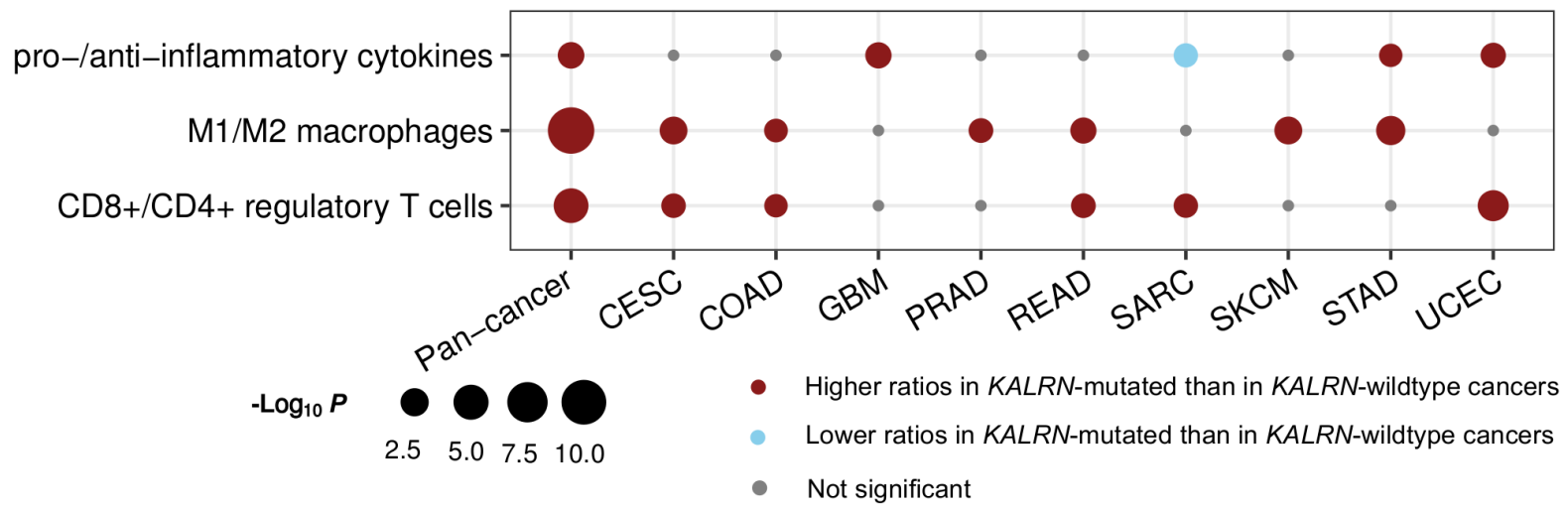

C

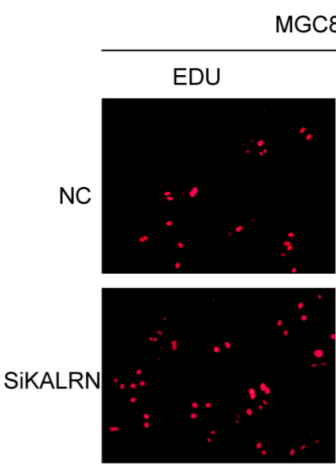

MGC803

DAPI

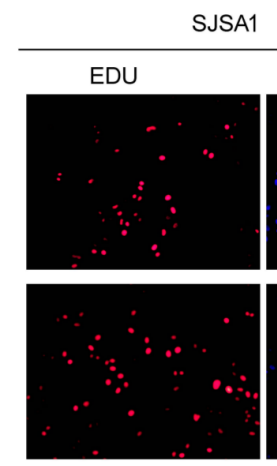

DAPI

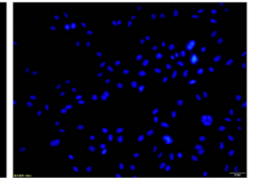

50pM
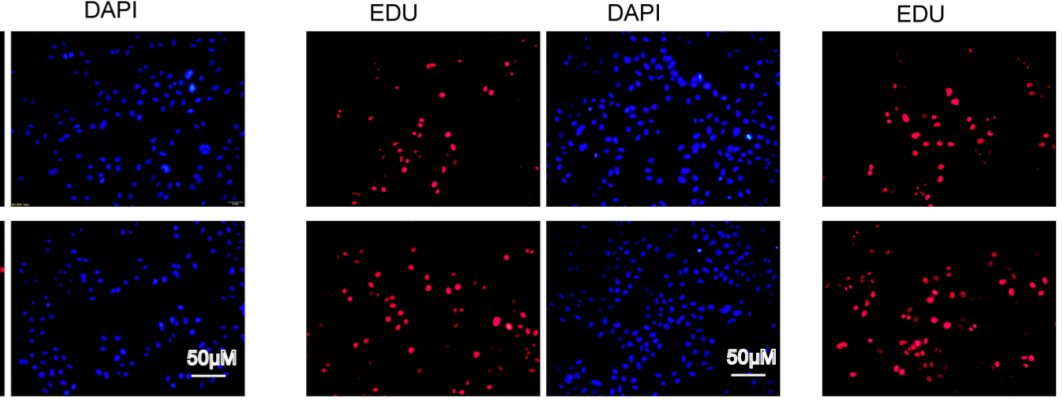

SW620
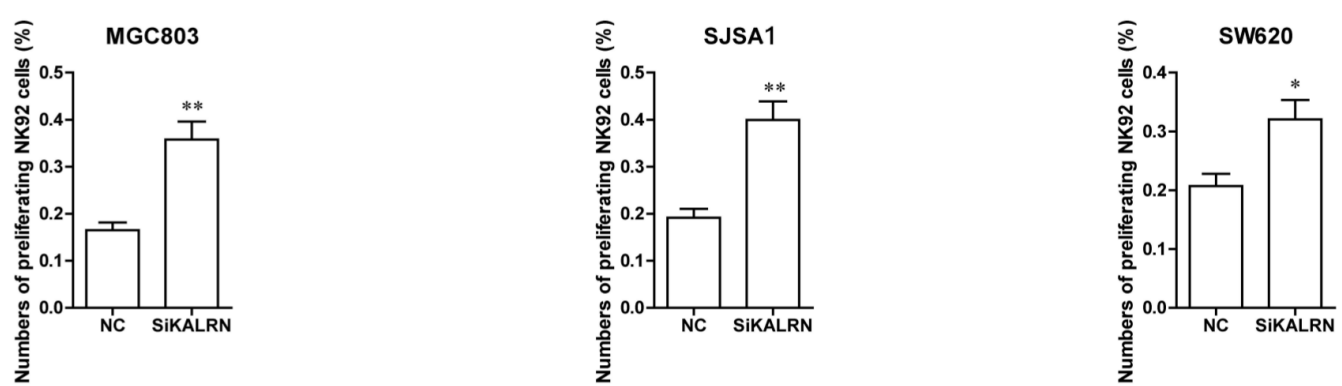

Figure 1 KALRN mutations promote antitumor immunity in cancer. (A) Three immune signatures (NK cells, CD8 +T cells and immune cytolytic activity) show higher enrichment levels in $K A L R N$-mutated than $K A L R N$-wildtype cancers in the pan cancer and in multiple individual cancer types (Mann-Whitney $U$ test, one tailed, $\mathrm{p}<0.05$ ). The enrichment levels of the immune signatures were evaluated by SsGSEA ${ }^{15}$ of their marker genes. (B) The ratios of immune-stimulatory/immune-inhibitory signatures are significantly higher in $K A L R N$-mutated than $K A L R N$-wildtype cancers in the pan cancer and in multiple individual cancer types (Mann-Whitney $U$ test, one tailed, $p<0.05$ ). The ratios are the mean expression levels of immune-stimulatory signature maker genes divided by the mean expression levels of immune-inhibitory signature maker genes. (C) NK cells cocultured with $K A L R N$-knockdown tumor cells display significantly stronger proliferation capacity than NK cells co-cultured with $K A L R N$-wildtype tumor cells, as evidenced by the EDU proliferation assay. Three human cancer cell lines (MGC803 (gastric cancer), SJSA1 (osteosarcoma) and SW620 (colon cancer)) and a murine cell line (B16F10 (melanoma)) were used in the in vitro experiments. (This also applies to the following figures). KALRN, kalirin RhoGEF kinase; ssGSEA, single-sample gene-set enrichment analysis; NK, natural killer; EDU, 5-Ethynyl-2'-deoxyuridine; DAPI, 6-diamidino-2-phenylindole. 

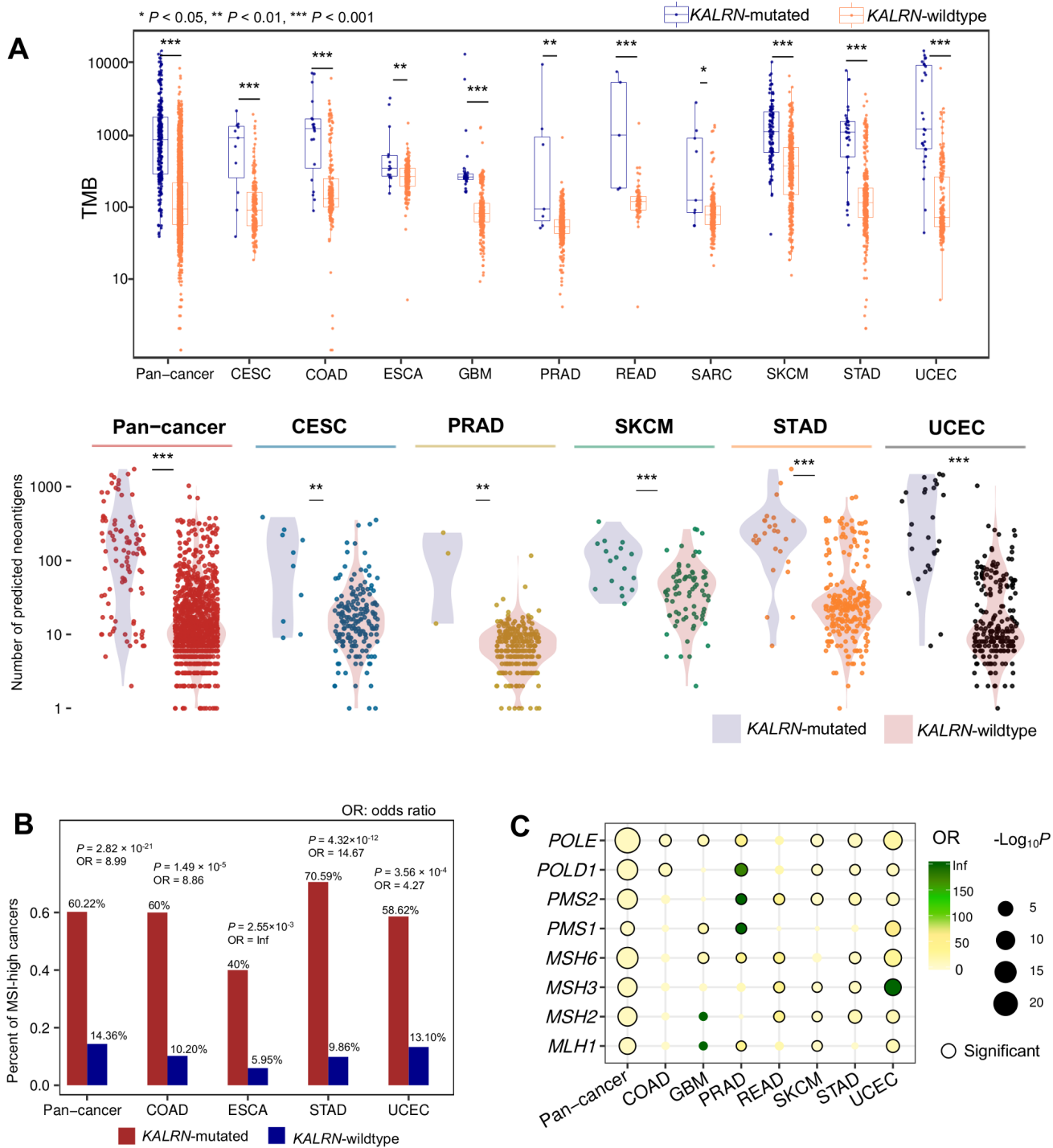

D

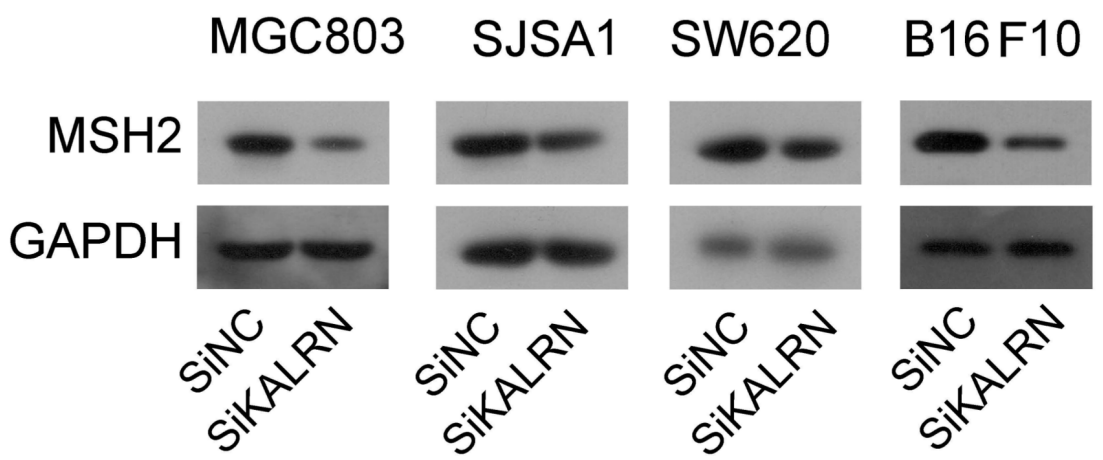

Figure 2 Associations of KALRN mutations with the TMB, neoantigens and microsatellite instability or DNA damage repair deficiency genomic properties in cancer. (A) KALRN-mutated cancers have significantly higher TMB and neoantigen loads than $K A L R N$-wildtype cancers in the pan cancer and in multiple individual cancer types (Mann-Whitney $\mathrm{U}$ test, one tailed, $\mathrm{p}<0.05$ ). TMB is defined as the total count of gene somatic mutations, and the neoantigens were predicted in reference. ${ }^{16}(\mathrm{~B}) \mathrm{MSI}-$ high cancers harbor more frequent mutations of KALRN than MSI-low/MSS cancers in the pan cancer and in four individual cancer types with a prevalent MSI subtype (Fisher's exact test, $\mathrm{p}<0.01$ ). (C) KALRN mutations likely co-occur with mutations of DNA damage repair pathway genes (Fisher's exact test, $\mathrm{p}<0.05$ ). (D) $K A L R N$-knockdown reduced MSH2 expression in tumor cells, as evidenced by Western blotting. GBM, glioblastoma multiforme; KALRN, kalirin RhoGEF kinase; TMB, tumor mutation burden; UCEC, uterine corpus endometrial carcinoma; ESCA, esophageal carcinoma; CESC, cervical squamous-cell carcinoma and endocervical adeno-carcinoma; COAD, colon adenocarcinoma; PRAD, prostate adenocarcinoma; SKCM, skincutaneous melanoma. 
MSH2, MSH3 and MSHO) and DNA replication and repair genes (POLD1 and POLE) in the pan cancer and in multiple individual cancer types (Fisher's exact test, $\mathrm{p}<0.05$ ) (figure $2 \mathrm{C}$ ). By analyzing the TCGA protein expression profiling datasets with MSH2 and MSH6 expression values available, we found that MSH2 and MSH6 were downregulated in $K A L R N$-mutated vs $K A L R N$-wildtype cancers in UCSC and that MSH6 was downregulated in $K A L R N$-mutated cancers in COAD (Student's t test, $\mathrm{p}<0.05$ ). Furthermore, in vitro experiments showed that MSH2 expression was reduced in $K A L R N$-knockdown relative to $K A L R N$-wildtype tumor cells (MGC803, SJSA1, SW620 and B16F10) (figure 2D). Overall, these results suggest a significant association between KALRN mutations and deficient DNA damage repair, which could explain the significantly increased TMB in KALRN-mutated cancers.

\section{Associations of the KALRN downstream target Rho GTPases with TMB, DNA damage repair and tumor immunity}

KALRN is a member of the Rho-GEF family that activates Rho GTPases by promoting GDP/GTP exchange. ${ }^{17}$ As a result, KALRN inactivation may compromise the activation of Rho GTPases. Indeed, KALRN expression was significantly downregulated in KALRN-mutated vs KALRNwildtype cancers in the pan cancer analysis (Student's $\mathrm{t}$ test, two tailed, $\mathrm{p}=1.11 \times 10^{-5}$ ), suggesting that KALRN mutations are inactivating mutations that impair KALRN activation. Network analysis with STRING ${ }^{18}$ showed that KALRN interacted with multiple members of the Rho GTPase family, including RAC1, RAC2, RAC3, RHOA, RHOB, RHOC, RHOD, RHOG and CDC42 (figure 3A). Moreover, we found that KALRN mutations likely co-occurred with mutations of these Rho GTPase genes in the pan cancer and in eight individual cancer types (Fisher's exact test, $\mathrm{p}<0.05$ ) (figure 3B). Previous studies demonstrated that Rho GTPases might play a role in regulating the DNA damage response. ${ }^{19}$ As expected, Rho GTPase gene-mutated cancers had a significantly higher TMB than Rho GTPase gene-wildtype cancers in the pan cancer and in eight individual cancer types (MannWhitney $\mathrm{U}$ test, one tailed, $\mathrm{p}<0.01$ ) (figure $3 \mathrm{C}$ ). Rho GTPase gene-mutated cancers exhibited more predicted neoantigens ${ }^{16}$ than Rho GTPase gene-wildtype cancers in the pan cancer and in five individual cancer types (Mann-Whitney $\mathrm{U}$ test, $\mathrm{p}<0.05$ ) (figure 3C). The mutations of Rho GTPase genes co-occurred with the mutations of DNA damage repair pathway genes (PMS1, PMS2, MLH1, MSH2, MSH3, MSH6, POLD1 and POLE) in the pan cancer and in multiple individual cancer types (Fisher's exact test, $\mathrm{p}<0.05$ ) (figure 3D). Moreover, the mutations of Rho GTPase genes more frequently occurred in MSI-H than in MSI-L/MSS cancers in the pan cancer and in two individual cancer types (STAD and COAD; Fisher's exact test, $\mathrm{p}<0.05$ ) (figure $3 \mathrm{E}$ ). Collectively, these results confirmed the critical role of Rho GTPases in mediating DNA damage repair pathways.
Because Rho GTPase deficiency may cause DNA damage repair deficiency that, in turn, would result in increased TMB and neoantigens, we expected that Rho GTPase gene-mutated cancers would display enhanced antitumor immune signatures. As expected, the three immune signatures (NK cells, $\mathrm{CD} 8+\mathrm{T}$ cells and ICA) were consistently stronger in Rho GTPase gene-mutated than Rho GTPase gene-wildtype cancers in the pan cancer (Mann-Whitney $\mathrm{U}$ test, one tailed, $\mathrm{p}<0.01$ ), as well as in diverse individual cancer types (Mann-Whitney $\mathrm{U}$ test, one tailed, $\mathrm{p}<0.05$ ) (figure $3 \mathrm{~F}$ ). Moreover, Rho GTPase gene-mutated cancers had significantly higher ratios of immune-stimulatory signatures to immune-inhibitory signatures $(\mathrm{CD} 8+/ \mathrm{CD} 4$ +regulatory $\mathrm{T}$ cells and M1/M2 macrophages) in the pan cancer and in diverse individual cancer types (Mann-Whitney U test, one tailed, $\mathrm{p} \leq 0.05$ ) (figure $3 \mathrm{~F}$ ).

Together, these data suggest that the significant correlations of KALRN mutations with increased TMB/neoantigens, deficient DNA damage repair pathways, and enhanced antitumor immune infiltrates could be consequences of the deregulation of the Rho GTPases targeted by KALRN.

\section{KALRN mutations correlate with elevated PD-L1 expression and favorable immunotherapy response}

We found that $P D-L 1$ showed significantly higher expression levels in $K A L R N$-mutated than KALRN-wildtype cancers in the pan cancer and in four individual cancer types (Student's t test, $\mathrm{p}<0.05$ ) (figure 4A). In vitro experiments showed that PD-L1 expression was significantly upregulated in KALRN-knockdown versus KALRN-wildtype tumor cells (MGC803, SJSA1, SW620 and B16F10) (figure 4B). These data suggest that PD-1/PD-L1-directed immunotherapy is likely to be more effective against $K A L R N$-mutated cancers because PD-L1 expression is a biomarker for an active response to anti-PD-1/PD-L1 immunotherapy. ${ }^{6}$ To prove this hypothesis, we investigated the association between KALRN mutations and ICB therapy response in five cancer cohorts receiving anti-PD-1/PD-L1/ CTLA-4 immunotherapy, namely the Allen cohort ${ }^{1}$ (melanoma), Hellmann cohort ${ }^{20}$ (lung cancer), Rizvi cohort $^{21}$ (lung cancer), Riaz cohort ${ }^{22}$ (melanoma) and Hugo cohort ${ }^{23}$ (melanoma). We found that KALRNmutated cancers had significantly higher response rates than $K A L R N$-wildtype cancers in these cohorts (37.04\% vs $10.96 \%$ in the Allen cohort, $45 \%$ vs $11.76 \%$ in the Hugo cohort, $80 \%$ vs $27.5 \%$ in the Riaz cohort, $100 \%$ vs $40.74 \%$ in the Rizvi cohort, and $70 \%$ vs $29.31 \%$ in the Hellmann cohort; Fisher's exact test, $\mathrm{p}<0.1$ ) (figure 4C). Because of the appreciably higher response rates, $K A L R N$-mutated cancers displayed a more favorable overall or progress-free survival tendency than $K A L R N$-mutated cancers (log-rank test, $\mathrm{p}=0.262,0.067,0.055,0.176$ and 0.047 for the Allen, Hugo, Riaz, Rizvi and Hellmann cohorts, respectively) (figure 4D). However, the correlation between KALRN 

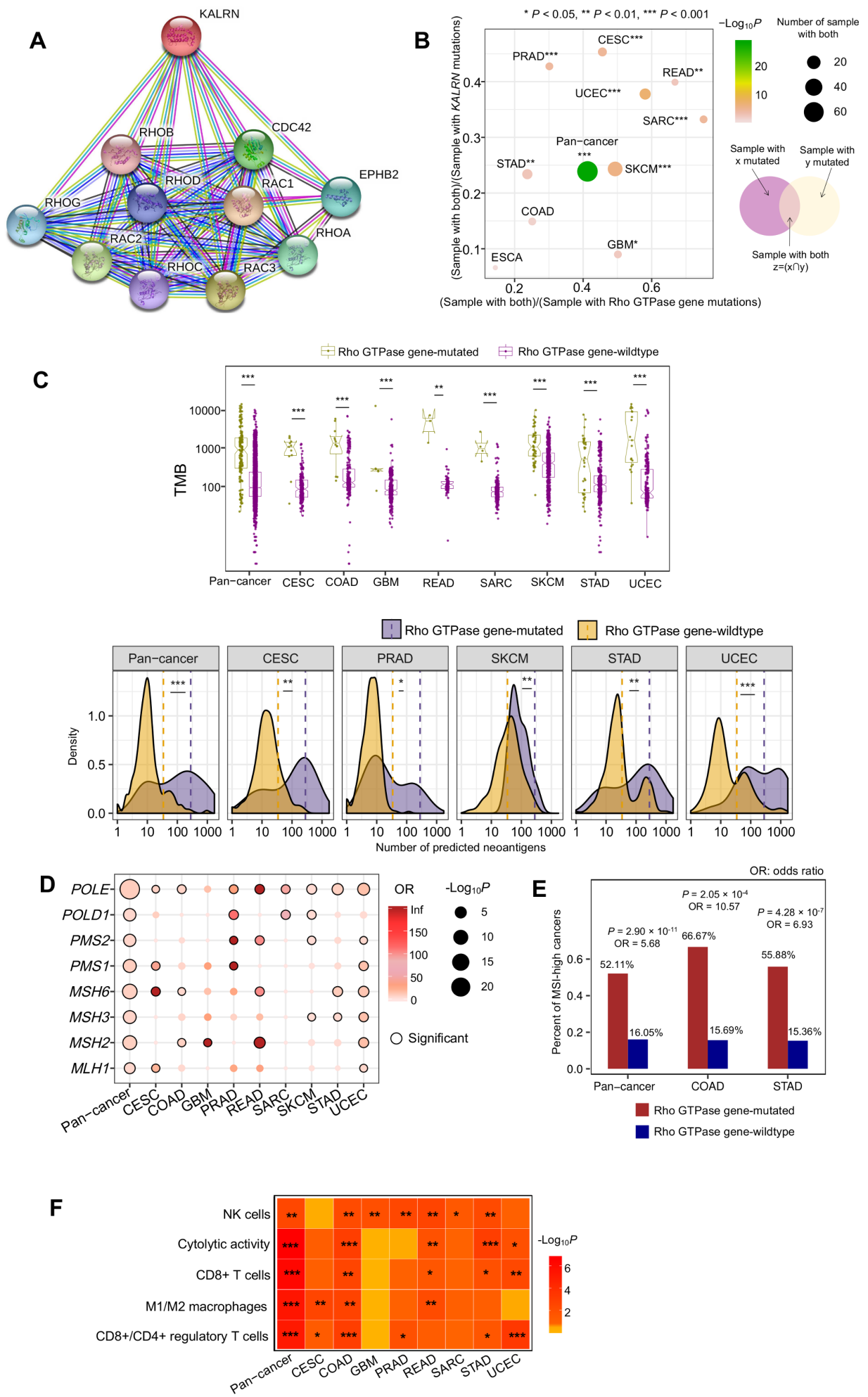

Figure 3 Associations of Rho GTPases with TMB, DNA damage repair pathways and tumor immunity. (A) KALRN interacts with multiple members of the Rho GTPase family, according to network analysis with STRING ${ }^{18}$ (B) KALRN mutations likely co-occur with Rho GTPase genes in the pan cancer and in multiple individual cancer types (Fisher's exact test, $p<0.05$ ). (C) Rho GTPase gene-mutated cancers have higher TMB and neoantigen loads than Rho GTPase gene-wildtype cancers in the pan cancer and in multiple individual cancer types (Mann-Whitney $U$ test, one tailed, $p<0.01$ ). (D) Mutations of the Rho GTPase genes likely co-occur with mutations of the DNA damage repair pathway genes in the pan cancer and in multiple individual cancer types (Fisher's exact test, $\mathrm{p}<0.05$ ). (E) The mutations of Rho GTPase genes more frequently occur in MSI-high than in MSI-low/MSS cancers in the pan cancer and in two individual cancer types (Fisher's exact test, $p<0.05$ ). (F) Rho GTPase genemutated cancers have stronger antitumor immune signatures than Rho GTPase gene-wildtype cancers in the pan cancer and in diverse individual cancer types (Mann-Whitney $\mathrm{U}$ test, one tailed, $\mathrm{p}<0.05$ ). GBM, glioblastoma multiforme; KALRN, kalirin RhoGEF kinase; TMB, tumor mutation burden; UCEC, uterine corpus endometrial carcinoma. 
A

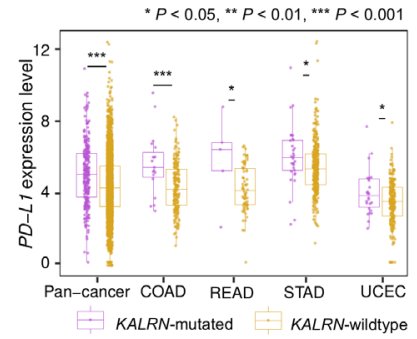

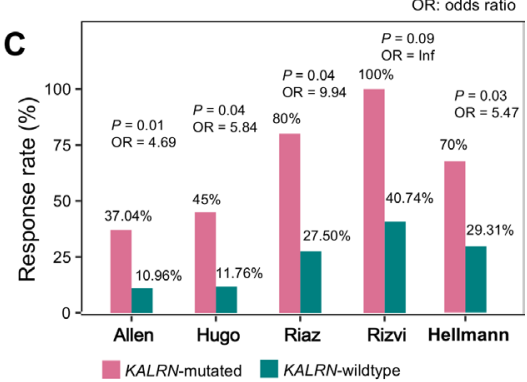

B

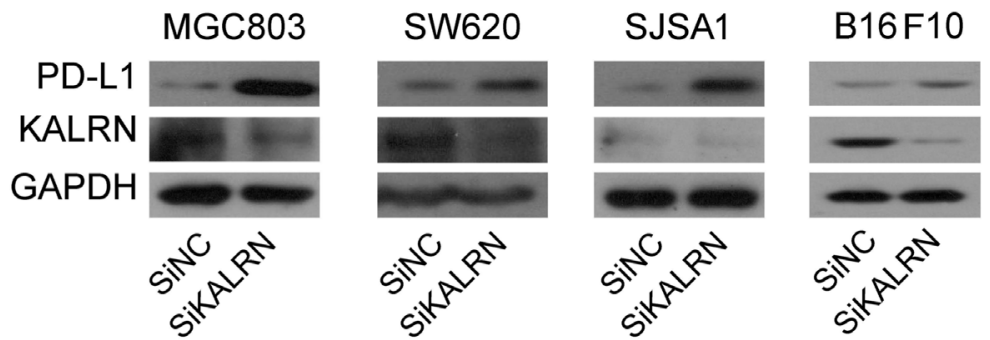

D
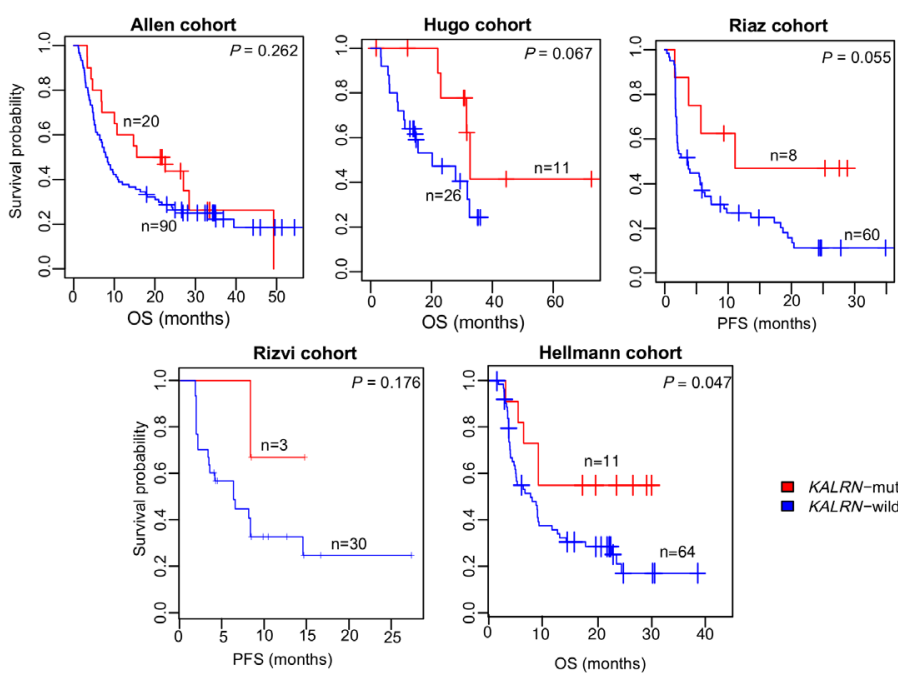

- KALRN-mutated

E
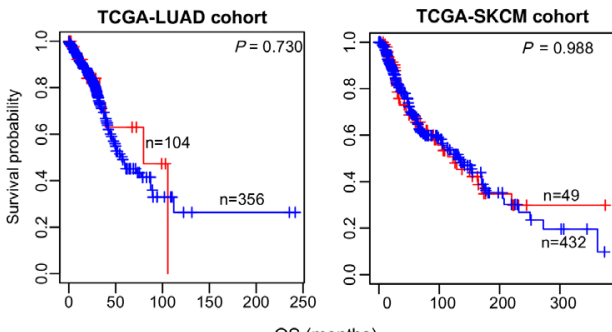

KALRN-mutated

- KALRN-wildtype

OS (months)

Figure 4 Associations of $K A L R N$ mutations with cancer immunotherapy response. (A) KALRN-mutated cancers display higher $P D$ - $L 1$ expression levels than $K A L R N$-wildtype cancers in the pan cancer and in multiple individual cancer types (Student's $\mathrm{t}$ test, two tailed, $\mathrm{p}<0.05)$. (B) The KALRN-knockdown increased PD-L1 expression in tumor cells, as evidenced by Western blotting. (C) KALRN-mutated cancers have higher response rates than KALRN-wildtype cancers in five cancer cohorts (the Allen cohort $^{1}$ (melanoma), Hellmann cohort ${ }^{20}$ (lung cancer), Rizvi cohort ${ }^{21}$ (lung cancer), Riaz cohort ${ }^{22}$ (melanoma) and Hugo cohort ${ }^{23}$ (melanoma)) receiving anti-PD-1/PD-L1/CTLA-4 immunotherapy. The Fisher's exact test $\mathrm{p}$ value and OR are shown for each cohort. (D) KALRN-mutated cancers show better overall survival (OS) or progress-free survival (PFS) tendencies than KALRNmutated cancers in the five cancer cohorts receiving anti-PD-1/PD-L1/CTLA-4 immunotherapy. (E) The association between KALRN mutations and OS is much less significant in TCGA melanoma and lung cancer cohorts without immunotherapy than in the five cancer cohorts receiving anti-PD-1/PD-L1/CTLA-4 immunotherapy. In (D) and (E), Kaplan-Meier survival curves are used to exhibit the survival time differences, and the log-rank test $p$ values are shown. CTLA-4, cytotoxic T-lymphocyte-associated protein 4; KALRN, kalirin RhoGEF kinase; PD-1, programmed cell death protein 1; PD-L1, programmed cell death 1 ligand; TCGA, The Cancer Genome Atlas; UCEC, uterine corpus endometrial carcinoma. 
mutations and overall survival was much less significant in TCGA melanoma or lung cancer cohorts not receiving immunotherapy (figure $4 \mathrm{E}$ ). These results indicate that the more favorable ICB therapy response in $K A L R N$-mutated cancer cohorts is attributed to the high antitumor immune infiltrates and PD-L1 expression in this subtype, suggesting that KALRN mutation is a predictive biomarker for an active response to ICB therapy.

\section{Validation by in vivo experiments}

To verify whether KALRN deficiency can enhance antitumor immune signatures and sensitization to ICB therapy, we performed in vivo experiments with mouse tumor models. We found that KALRN loss markedly increased B16F10-tumor volume and progression (figure 5A). KALRN-depleted tumors displayed significant increases in CD8 + T cell infiltration (figure 5B) and PD-L1 expression (figure 5C); this is consistent with the previous results. Moreover, in KALRN-depleted tumors, the expression levels of IFN- $\gamma$ and TNF- $\alpha$ were significantly reduced (figure 5D), indicating that the activity of tumor-infiltrating CD8 +lymphocytes is impaired in $K A L R N$-depleted tumors. The expression levels of PD-1 and LAG3 were also markedly enhanced in KALRNdeficient tumors (figure 5E,F), suggesting that KALRN deficiency promotes CD8 + Tcell exhaustion. Likewise, $K A L R N$-depleted tumors displayed significant increases in NK cell infiltration (figure 5G) but reduced activity of tumor-infiltrating NK lymphocytes (figure $5 \mathrm{H}$ ). Moreover, the volume of the KALRN-depleted tumors markedly decreased relative to that of the KALRN-wildtype tumors after use of BMS-1, a small-molecule inhibitor of PD-1/PD-L1 (figure 5I); this result indicates higher sensitivity of the KALRN-depleted tumor to the PD-1/PD-L1 inhibitor. This is consistent with the previous finding that $K A L R N$ mutations correlated positively with the response to ICB therapy. Furthermore, although the activities of tumor-infiltrating CD8 +and NK lymphocytes were lower in $K A L R N$-depleted than $K A L R N$-wildtype tumors, they were significantly higher in $K A L R N$-depleted tumors after treatment with BMS-1 (figure 5J,K). These data suggest that the PD-1/PD-L1 inhibitor inhibits the exhaustion of tumor-infiltrating CD8+ and NK lymphocytes in KALRNdepleted tumors, thereby promoting the killing of tumor cells by the immune system.

\section{DISCUSSION}

For the first time, we report the significant role of KALRN mutations in promoting anti-tumor immunity and immunotherapeutic vulnerabilities in diverse cancer types. KALRN mutations can enhance antitumor immunity because they increase the tumor mutation load to create more neoantigens as a result of DNA damage repair deficiency. KALRN-mutated cancers express more PD-L1, in addition to the increased anti-tumor immune infiltrates. Because both tumor-infiltrating lymphocyte density and PD-L1 expression are important factors in determining ICB therapy responsiveness, ${ }^{6}{ }^{24}$ it would be reasonable for $K A L R N$-mutated cancers to exhibit increased susceptibility to ICB therapy. This inference was evidenced in five cancer cohorts receiving ICB therapy (figure 4C,D).

We investigated the underlying mechanism of $K A L R N$ mutations in enhancing antitumor immunity and immunotherapy response (figure 6). The significant association between KALRN mutations and heightened antitumor immunity may be attributed to the fact that KALRN inactivation compromises the function of the target Rho GTPases in regulating DNA damage repair pathways. Previous studies have revealed the function of Rho GTPases in modulating DNA damage response. ${ }^{24-26}$ In this study, we demonstrated a significant correlation between Rho GTPases and DNA damage repair pathways (figure 3C-E). Rho GTPases are involved in a wide variety of cellular functions and oncogenic processes, including the cell cycle, cell death, cell migration, cell polarity and cell adhesion. ${ }^{27}$ Notably, previous studies have shown that inhibition of Rho GTPases can promote an antitumor immune response by increasing the ICA of cytotoxic CD8 + T lymphocytes. ${ }^{27}$ This finding is in line with our results showing that the antitumor immune signatures grew stronger in cancers harboring mutations of Rho GTPase genes (figure 3F). Interestingly, we found that the cancers with mutations of Rho GTPase genes overexpressed $P D-L 1$ in the pan cancer and multiple individual cancer types (Mann-Whitney $\mathrm{U}$ test, one tailed, $\mathrm{p}<0.05)$. This suggests that mutations of Rho GTPase genes may favor the ICB therapy response. Indeed, we found that cancers harboring mutations of Rho GTPase genes showed higher ICB therapy response rates than those without such mutations in the five cancer cohorts receiving ICB therapy $(42.86 \%$ vs $22.78 \%$ in the Allen cohort, $70 \%$ vs $48.15 \%$ in the Hugo cohort, $83.33 \%$ vs $25.64 \%$ in the Riaz cohort, $100 \%$ vs $45 \%$ in the Rizvi cohort, and $50 \%$ vs $28.26 \%$ in the Hellmann cohort). Nevertheless, logistic regression analyzes showed that the KALRN mutation was a significant positive predictor for the immunotherapy response in three of the five cancer cohorts after correction for the Rho GTPase gene mutation $(\mathrm{p}<0.05)$ (online supplemental figure $\mathrm{S} 1 \mathrm{~A}$ ). In addition, the KALRN mutation was a significant positive predictor for the immune signature scores in TCGA pan cancer after correcting for the Rho GTPase gene mutation $(\mathrm{p}<0.001)$ (online supplemental figure $\mathrm{S} 1 \mathrm{~B}$ ). These results indicate that KALRN mutations may promote antitumor immunity and immunotherapy response via other approaches besides the upstream regulation of Rho GTPases. As expected, the Rho GTPase gene mutation was also a positive predictor for the immunotherapy response and immune signature scores, whereas its $\beta$ coefficient was smaller than that of the KALRN mutation 


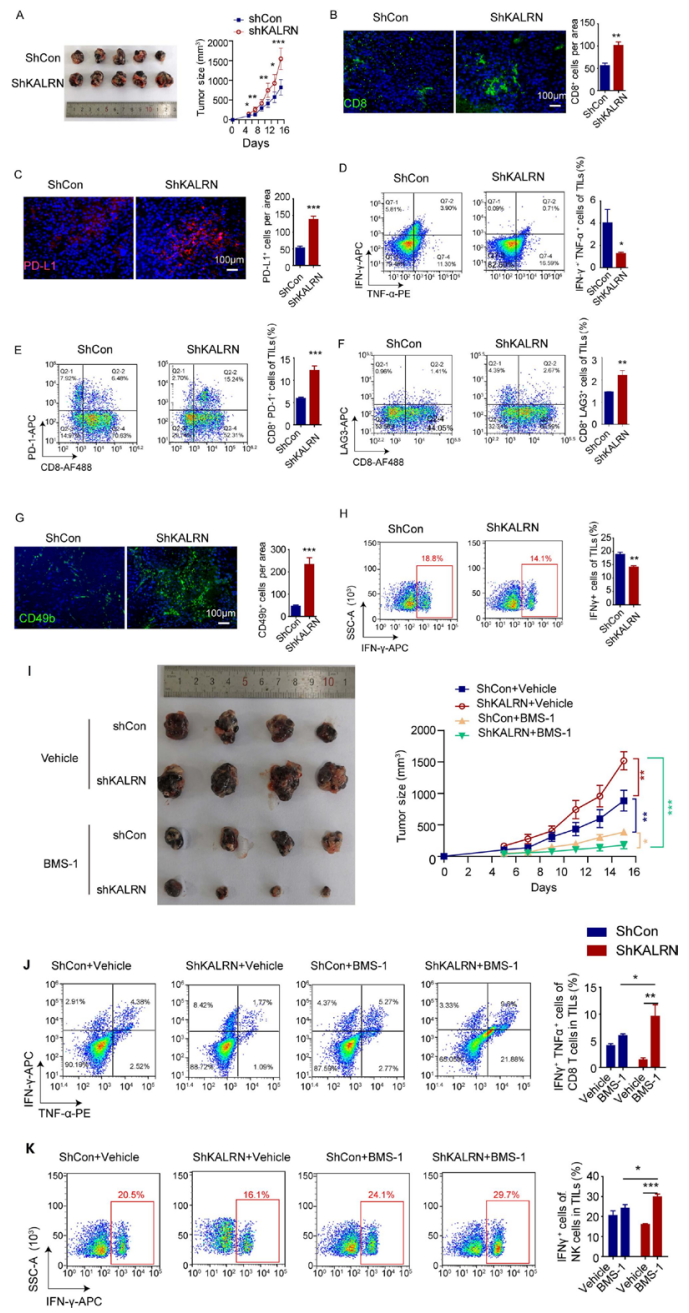

Figure $5 K A L R N$-deficient tumors display increased tumor-infiltrating lymphocytes, expression of immune checkpoint molecules, and sensitization to immune checkpoint inhibitors. (A-H) B16F10 tumor cells stably transfected with ShCon or ShKALRN lentivirus were subcutaneously injected into C57BL6 mice. The tumor volumes were measured every 2 days from the fifth day to the fifteenth. After sacrifice of the mice, the tumor tissues were isolated for immunofluorescence (IF) or for isolation of TILs. (A) Representative tumor images of mice bearing ShCon and ShKALRN tumors. The graph shows the change in tumor size in the mice over time. Data represent mean $\pm S E$. ( $n=5$ for each group, two-tailed test, compared against the ShCon group, ${ }^{*} \mathrm{p}<0.05,{ }^{* *} \mathrm{p}<0.01,{ }^{* \star *} \mathrm{p}<0.001$ ). (B) CD8 IF staining in B16F10 orthotopic tumors and $\mathrm{H}$-score analysis ( $\mathrm{n}=5$ for each group, two-tailed t test, compared against the ShCon group, $\left.{ }^{\star *} \mathrm{p}<0.01\right)$. (C) PD-L1 IF staining in B16F10 orthotopic tumors and Hscore analysis $\left(n=5\right.$ for each group, two-tailed t test, compared against the ShCon group, $\left.{ }^{* *} p<0.01\right)$. (D) TILs were stained with CD3-BV421, CD8-AF-488, TNF- $\alpha$-PE, and IFN- $\gamma$-APC; they were then analyzed by flow cytometry. Lymphocytes were gated according to forward scatter and side scatter. CD3 and CD8 staining was used to identify CD8 T cells. A comparison of TNF- $\alpha$ and IFN- $\gamma$ expression on CD8 T cells from TILs in mice is shown ( $n=5$ for each group, two-tailed test, compared against the ShCon group, $\left.{ }^{*} \mathrm{p}<0.05\right)$. (E) TILs were stained with CD3-BV421, CD8-AF-488, and PD-1-APC; they were then analyzed by flow cytometry. PD-L1 expression on CD8 T cells from TILs in mice is shown ( $n=5$ for each group, two-tailed t test, compared against the ShCon group, $\left.{ }^{* \star *} p<0.001\right)$. (F) TILs were stained with CD3-BV421, CD8-AF-488, and LAG3-APC; they were then analyzed by flow cytometry. LAG3 expression on CD8 T cells from TILs in mice is shown ( $n=5$ for each group, two-tailed t test, compared against the ShCon group, ${ }^{\star *} \mathrm{p}<0.01$ ). (G) CD49b IF staining in B16F10 orthotopic tumors and $\mathrm{H}$-score analysis ( $\mathrm{n}=5$ for each group, two-tailed t test, compared against the ShCon group, $\left.{ }^{\star * *} \mathrm{p}<0.001\right)$. (H) TILs were stained with CD3-BV421, CD49bPE and IFN- $\gamma$-APC; they were then analyzed by flow cytometry. CD3- and CD49b+staining was used to identify NK cells. A comparison of IFN- $\gamma$ expression on NK cells from TILs in mice is shown ( $n=5$ for each group, two-tailed t test, compared against the ShCon group, $\left.{ }^{\star \star \star} \mathrm{p}<0.001\right)$. (I-K) KALRN-deficient tumors formed by subcutaneous injection of B16F10 cells, as mentioned in A-H. ShCon and ShKALRN tumor-bearing mice were divided into vehicle groups and BMS-1 groups. The vehicle groups were treated with solvent, whereas BMS-1 group mice were treated with BMS-1. (I) Representative tumor images of mice are shown on the left. The graph on the right shows the change in tumor size in the mice over time. Data represent mean $\pm S E$. $(n=5$ for each group, one-way ANOVA, $\left.{ }^{*} p<0.05,{ }^{* *} p<0.01,{ }^{* * *} p<0.001\right)$. (J) TILs were treated as described in D). Comparisons of TNF- $\alpha$ and IFN- $\gamma$ expression on CD8 T cells from TILs in mice are shown ( $n=5$ for each group, one-way ANOVA, ${ }^{*} p<0.05$, $\left.{ }^{* *} p<0.01\right)$. (K) TILs were treated as described in $\mathrm{H}$. Comparisons of IFN- $\gamma$ expression on NK cells from TILs in mice are shown ( $n=5$ for each group, one-way ANOVA, ${ }^{*} \mathrm{p}<0.05,{ }^{* *} \mathrm{p}<0.001$ ). ANOVA, analysis of variance IFN $-\gamma$, interferon- $\gamma$; KALRN, kalirin RhoGEF kinase; TNF- $\alpha$, tumor necrosis factor- $\alpha$; TILs, tumor-infiltrating lymphocytes; APC, Allophycocyanin. 


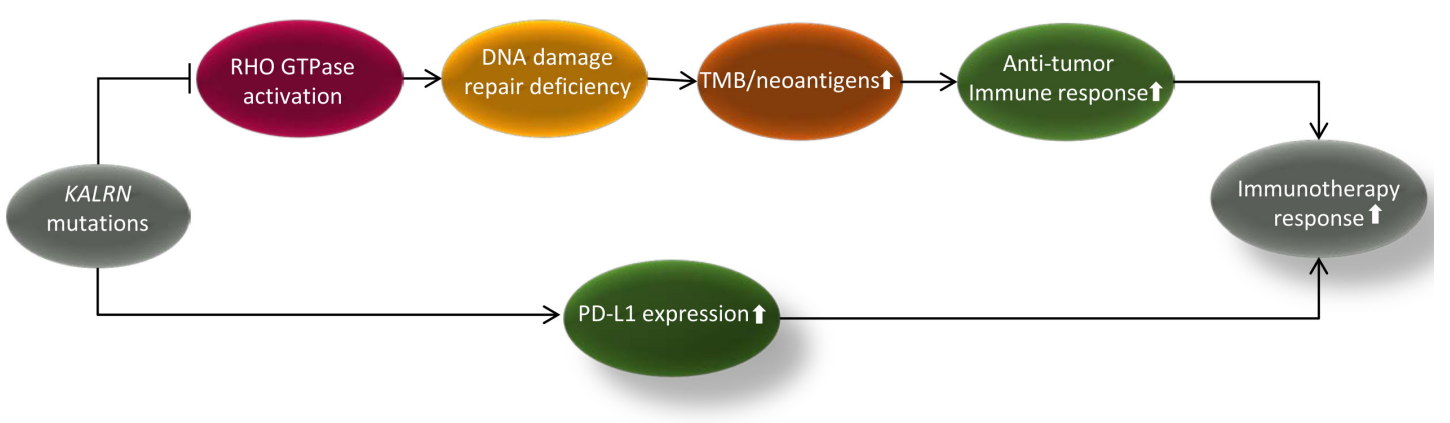

Figure 6 The mechanism by which KALRN mutations promote antitumor immunity and immunotherapy response in cancer. KALRN, kalirin RhoGEF kinase; PD-L1, programmed cell death 1 ligand.

in almost all cases (online supplemental figure S1). In addition, we found that the association between the Rho GTPase gene mutation and survival was less significant in the $K A L R N$-wildtype cancer subset than in all cancers in three of the cancer cohorts receiving ICB therapy (online supplemental figure S2). Overall, these results indicate that Rho GTPase mutations may not correlate independently with antitumor immunity and immunotherapy response but rather in a KALRNdependent manner.

A limitation of this study is that the sample sizes were not sufficiently large in the cancer cohorts receiving immunotherapy. To overcome this limitation, we need to validate our findings by using more significant numbers of cancer samples with the immunotherapy response data available in the future.

\section{CONCLUSIONS}

The KALRNmutation correlates with enhanced antitumor immunity and immunotherapy response and, thus, is a predictive biomarker for the response to immunotherapy.

Acknowledgements We thank Ms. Yin He for help in performing the experiments.

Contributors ML performed experiments and data analyses, and edited the manuscript. YM performed experiments and data analyses, and edited the manuscript. YZ performed experiments. QL performed experiments. CC performed experiments. LQ performed experiments. XW conceived this study, designed the methods and wrote the manuscript. All the authors read and approved the final manuscript.

Funding This work was supported by the China Pharmaceutical University (grant number 3150120001 to XW) and Jiangsu Province Graduate Research and Innovation Program Project (grant number KYCX20_0658 to ML).

Competing interests None declared.

Patient consent for publication Not required.

Provenance and peer review Not commissioned; externally peer reviewed.

Data availability statement Data are available in a public, open access repository. Data are available on reasonable request. All data relevant to the study are included in the article or uploaded as online supplemental information. The datasets for the 10 TCGA cancer cohorts can be downloaded from the genomic data commons data portal (https://portal.gdc.cancer.gov/). The 10 cancer cohorts included cervical squamous-cell carcinoma and endocervical adeno-carcinoma (CESC), colon adenocarcinom (COAD), Esophageal Carcinoma (ESCA), Glioblastoma Multiforme (GBM), Prostate Adenocarcinoma (PRAD), Rectum Adenocarcinoma (READ), Sarcoma (SARC), Skincutaneous Melanoma (SKCM), Stomach Adenocarcinoma (STAD), and Uterine Corpus Endometrial Carcinoma (UCEC). For each cancer type, we obtained its gene somatic mutations, RNA-Seq gene expression profiles, protein expression profiles, and clinical data. For pan cancer, we downloaded the related datasets from UCSC (https://xenabrowser.net/datapages/). We obtained the data for predicted neoantigens in the TCGA cancer cohorts from the publication [14], in which a total of 6 cancer types (CESC, PRAD, GBM, STAD, SKCM, UCEC) had predicted neoantigen data available. For the four cancer cohorts receiving anti-PD-1/PD-L1/ CTLA-4 immunotherapy (Allen cohort, Hugo cohort, Riaz cohort, Rizvi cohort, and Hellmann cohort), we obtained their genomics and clinical data from the associated publications. A summary of these datasets is shown in online supplemental table S1.

Supplemental material This content has been supplied by the author(s). It has not been vetted by BMJ Publishing Group Limited (BMJ) and may not have been peer-reviewed. Any opinions or recommendations discussed are solely those of the author(s) and are not endorsed by BMJ. BMJ disclaims all liability and responsibility arising from any reliance placed on the content. Where the content includes any translated material, BMJ does not warrant the accuracy and reliability of the translations (including but not limited to local regulations, clinical guidelines, terminology, drug names and drug dosages), and is not responsible for any error and/or omissions arising from translation and adaptation or otherwise.

Open access This is an open access article distributed in accordance with the Creative Commons Attribution Non Commercial (CC BY-NC 4.0) license, which permits others to distribute, remix, adapt, build upon this work non-commercially, and license their derivative works on different terms, provided the original work is properly cited, appropriate credit is given, any changes made indicated, and the use is non-commercial. See http://creativecommons.org/licenses/by-nc/4.0/.

\section{ORCID iD}

Xiaosheng Wang http://orcid.org/0000-0002-7199-7093

\section{REFERENCES}

1 Van Allen EM, Miao D, Schilling B, et al. Genomic correlates of response to CTLA-4 blockade in metastatic melanoma. Science 2015;350:207-11.

2 Bauml J, Seiwert TY, Pfister DG, et al. Pembrolizumab for platinumand Cetuximab-Refractory head and neck cancer: results from a single-arm, phase II study. J Clin Oncol 2017;35:1542-9.

3 Herbst RS, Baas P, Kim D-W, et al. Pembrolizumab versus docetaxel for previously treated, PD-L1-positive, advanced non-small-cell lung cancer (KEYNOTE-010): a randomised controlled trial. Lancet 2016;387:1540-50.

4 Tomita Y, Fukasawa S, Shinohara N, et al. Nivolumab versus everolimus in advanced renal cell carcinoma: Japanese subgroup analysis from the CheckMate 025 study. Jpn J Clin Oncol 2017;47:639-46.

5 Ribas A, Wolchok JD. Cancer immunotherapy using checkpoint blockade. Science 2018;359:1350-5.

6 Patel SP, Kurzrock R. PD-L1 expression as a predictive biomarker in cancer immunotherapy. Mol Cancer Ther 2015;14:847-56.

7 Goodman AM, Kato S, Bazhenova L, et al. Tumor mutational burden as an independent predictor of response to immunotherapy in diverse cancers. Mol Cancer Ther 2017;16:2598-608.

8 Dong Z-Y, Zhong W-Z, Zhang X-C, et al. Potential Predictive Value of TP53 and KRAS Mutation Status for Response to PD-1 Blockade Immunotherapy in Lung Adenocarcinoma. Clin Cancer Res 2017;23:3012-24. 
9 Li L, Li M, Jiang Z, et al. ARID1A mutations are associated with increased immune activity in gastrointestinal cancer. Cells 2019;8:678

10 Penzes P, Johnson RC, Kambampati V, et al. Distinct roles for the two Rho GDP/GTP exchange factor domains of Kalirin in regulation of neurite growth and neuronal morphology. J Neurosci 2001;21:8426-34.

11 Tate JG, Bamford S, Jubb HC, et al. Cosmic: the Catalogue of somatic mutations in cancer. Nucleic Acids Res 2019;47:D941-7.

12 Sun Q, Li M, Wang X. The cancer omics atlas: an integrative resource for cancer omics annotations. BMC Med Genomics 2018;11:63.

13 Grønhøj C, Jensen DH, Agander T, et al. Deep sequencing of human papillomavirus positive loco-regionally advanced oropharyngeal squamous cell carcinomas reveals novel mutational signature. BMC Cancer 2018;18:640.

14 Xia J, Jia P, Hutchinson KE, et al. A meta-analysis of somatic mutations from next generation sequencing of 241 melanomas: a road map for the study of genes with potential clinical relevance. Mol Cancer Ther 2014;13:1918-28.

15 Hänzelmann S, Castelo R, Guinney J. GSVA: gene set variation analysis for microarray and RNA-Seq data. BMC Bioinformatics 2013;14:7.

16 Rooney MS, Shukla SA, Wu CJ, et al. Molecular and genetic properties of tumors associated with local immune cytolytic activity. Cell 2015;160:48-61.

17 Kratzer M-C, England L, Apel D, et al. Evolution of the Rho guanine nucleotide exchange factors Kalirin and trio and their gene expression in Xenopus development. Gene Expr Patterns 2019;32:18-27.

18 Szklarczyk D, Gable AL, Lyon D, et al. String v11: protein-protein association networks with increased coverage, supporting functional discovery in genome-wide experimental datasets. Nucleic Acids Res 2019;47:D607-13.

19 Fritz G, Henninger C. Rho GTPases: novel players in the regulation of the DNA damage response? Biomolecules 2015;5:2417-34.

20 Hellmann MD, Nathanson T, Rizvi H, et al. Genomic features of response to combination immunotherapy in patients with advanced non-small-cell lung cancer. Cancer Cell 2018;33:843-52.

21 Rizvi NA, Hellmann MD, Snyder A, et al. Cancer immunology. Mutational landscape determines sensitivity to PD-1 blockade in non-small cell lung cancer. Science 2015;348:124-8.

22 Riaz N, Havel JJ, Makarov V, et al. Tumor and microenvironment evolution during immunotherapy with nivolumab. Cell 2017:171:e16:934-49.

23 Hugo W, Zaretsky JM, Sun L, et al. Genomic and transcriptomic features of response to anti-PD-1 therapy in metastatic melanoma. Cell 2016;165:35-44.

24 Zito Marino F, Ascierto PA, Rossi G, et al. Are tumor-infiltrating lymphocytes protagonists or background actors in patient selection for cancer immunotherapy? Expert Opin Biol Ther 2017;17:735-46.

25 Sarrabayrouse G, Pich C, Teiti I, et al. Regulatory properties of statins and Rho GTPases prenylation inhibitiors to stimulate melanoma immunogenicity and promote anti-melanoma immune response. Int $J$ Cancer 2017:140:747-55.

26 Hodge RG, Ridley AJ. Regulating Rho GTPases and their regulators. Nat Rev Mol Cell Biol 2016;17:496-510.

27 Sarrabayrouse G, Pich C, Moriez R, et al. Melanoma cells treated with GGTI and IFN-gamma allow murine vaccination and enhance cytotoxic response against human melanoma cells. PLoS One 2010;5:e9043. 